\title{
Silencing of Mannitol Transporter Gene In Phelipanche Aegyptiaca Reduces The Parasite Development On The Host Root
}

Vinay Kumar Bari ( $\nabla$ vinay.bari@cup.edu.in )

Agricultural Research Organization (ARO) https://orcid.org/0000-0002-5670-3798

Jackline Abu Nassar

Agricultural Research Organization (ARO)

Radi Aly

Agricultural Research Organization (ARO)

\section{Short Report}

Keywords: Virus induced gene silencing (VIGS), Strigolactone, Plant parasitic weed, Mannitol transport

Posted Date: October 26th, 2021

DOl: https://doi.org/10.21203/rs.3.rs-981546/v1

License: (1) This work is licensed under a Creative Commons Attribution 4.0 International License.

Read Full License 
1 Silencing of mannitol transporter gene in Phelipanche aegyptiaca reduces the parasite

2 development on the host root

3 Vinay Kumar Bari ${ }^{1,2} *$, Jackline Abu Nassar ${ }^{1} \& \operatorname{Radi}$ Aly ${ }^{1 *}$

$4 \quad{ }^{1}$ Department of Plant Pathology and Weed Sciences, Newe Yaar Research Station, Agricultural

5 Research Organization (ARO), Israel.

$6 \quad{ }^{2}$ Department of Biochemistry, Central University of Punjab, VPO-Ghudda, Bathinda, India.

$7 *$ Correspondence

8 (Email: radi@volcani.agri.gov.il) https://orcid.org/0000-0002-6038-9586

9 (Email: vinay.bari@ cup.edu.in) https://orcid.org/0000-0002-5670-3798

10

Conflict of interest: All the authors declared that there is no potential conflict of interest. 
Abstract:

Parasitic weed Phelipanche aegyptiaca, is an obligate plant parasite which causes severe damage to host crops. Agriculture crops mainly belong to Brassicaceae, Leguminosae, Cruciferae and Solanaceae plant families affected by this parasitic weed which leads to devastating loss to crops yield and economic growth. This root specific parasitic plant is not able to complete its life-cycle without a suitable host and is totally dependent on the host plant for organic nutrients. Therefore, flow of organic solute molecules towards parasites is essential for the survival of the parasitic weed and disturbance in this network modulated host-parasitic interaction, which induces resistance in host against these parasitic weeds. To develop host resistance to this parasitic weed, we silenced selected prominent genes associated with $P$. aegyptiaca using virus-induced gene silencing (VIGS) methods. Our results demonstrated that the total number of parasite tubercle attached to root of the host plant Nicotiana benthamiana significantly reduced upon silencing of $P$. aegyptiaca specific gene which encodes mannitol transporter, however silencing of other gene(s), have no significant effects. Thus, our study indicates that mannitol transport plays an important role in host-parasite interaction and silencing of this gene causes development of host resistance against this parasite.

Keywords: Virus induced gene silencing (VIGS), Strigolactone, Plant parasitic weed, Mannitol transport 
The Phelipanche is an obligate root specific parasitic plant belonging to the Orobanchaceae family that represents a great challenge to almost all economically important crops and agriculture economy $[1,2]$. Due to lack of functional chloroplast these parasites are not able to synthesize organic solute and totally depend on the host plant for their survival [3]. The germination of this parasite seed is induced by a chemical molecule known as strigolactone (SL) exuded by the host plant into the rhizosphere $[4,5]$. After germination a special kind of interconnection between host root tissue and parasitic plant is established called as haustorium, and it is the main characteristic feature of parasitic plant that withdraws organic solute nutrient from the host towards parasites [6]. Haustorium helps parasites in withdrawal of organic nutrient molecules from the host, hence potentially damaging the host crop and greatly reducing the yields in terms of quality and quantity $[7,8]$. However, the nature of the solute molecules and its role in host-parasite interaction is less explored in P. aegyptiaca. Previous studies reported that during the initial phase of host-parasite attachment major alteration in metabolism occurs [9], and transported amino acids and sugars are two organic solute molecules that are mainly altered in the parasite during establishment of root connection with the host $[10,11]$. Recent comparative transcriptomic study revealed core parasitic genes in P.aegyptiaca, which were involved in interaction with host plants and the transcript level of these genes were altered upon parasitic infections [12]. Since these parasitic genes which are involved in host-parasite interaction could determine the nature of establishment and failure in these networks will lead to development of resistance, hence we have silenced these selected parasitic gene(s), which function in host-parasite interactions. The aim of our study is to evaluate the effectiveness of host resistance after silencing of these genes involved in hostparasite interaction. To degrade the mRNA transcript of these genes, we used tobacco rattle virus mediated gene silencing approaches [14] in $P$. aegyptiaca and demonstrated that the 
mannitol transporter gene upon silencing significantly reduces the development of P.aegyptiaca on host plant $N$. benthamiana.

\section{Results:}

Based on the Expression Sequence Tags data for the P. aegyptiaca deposited in Parasitic Plant Genome Project (PPGP), we extracted and confirmed the existence of $P$. aegyptiaca gene(s) encoding for mannitol transporter (PaMNT1), methionine synthase (PaMET1), sucrose transporter (PaSUT1), E3 ubiquitin ligase (PaUBQ3), oligopeptide transporter (PaOPT1), phosphate transporter $(\mathrm{PaPHTl})$, pleiotropic drug resistance $(\mathrm{PaPDR}$ ), CLP protease (PaCLP1), glutamate receptor (PaGLR1) and more axillary growth 2 (PaMAX2), which were shown to be transcriptionally altered during in host-parasite interaction [12]. Furthermore, to silence these selected genes of P.aegyptiaca, we introduce suitable gene specific DNA fragments (around 200bp for each gene) into Virus-Induced Gene Silencing (VIGS) construct [13]. To avoid putative silencing of the host genes, non-homologous DNA sequences of the targeted gene(s), that differ between host and the parasite, was chosen. The selected target regions of your favourite gene from $P$. aegyptiaca $(P a Y F G)$ were cloned in a VIGS system (pTRV2) using suitable restriction sites BamHI and $\mathrm{XbaI}$ (Fig. 1a). Recombinant clones were identified by diagnostic PCR using primer outside the ligated DNA sequence and further confirmed by Sangers DNA sequencing. $p T R V 2-P a Y F G$, a positive plasmid, was transformed in Agrobacterium tumefaciens strain EHA105, which is extensively used in plant transformation. Host plant $N$. benthamiana (3-4 leaves stage) were grown in the greenhouse and after 2 weeks, they were transferred to new pots containing P.aegyptiaca seeds $(15 \mathrm{mg} / \mathrm{kg}$ soil). The host plants were agroinfiltrated with A. tumefaciens EHA105 containing the recombinant $p T R V 2: P a Y F G$ and $p T R V 1$ according to the method described previously. To confirm the A. tumefaciens EHA105 mediated transformation and accumulation of $p T R V 1$ and 
$\mathbf{a}$

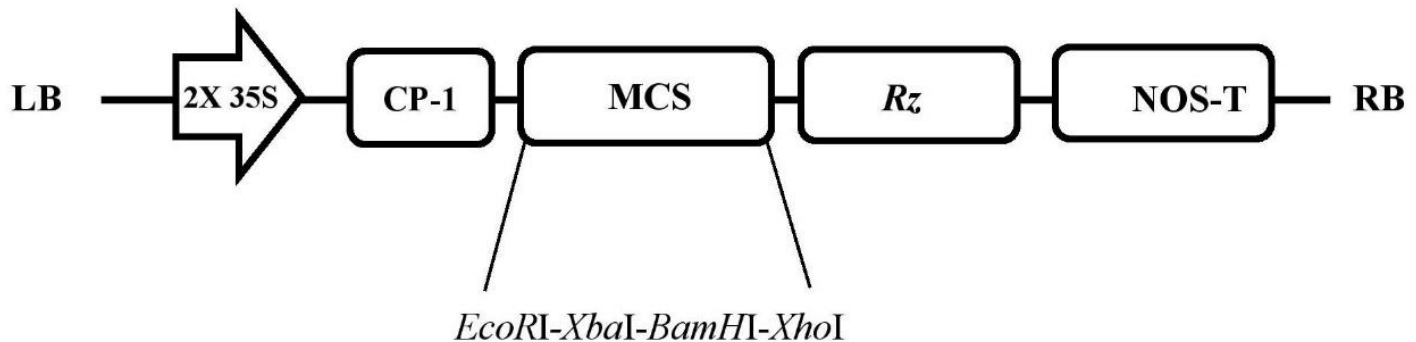

b

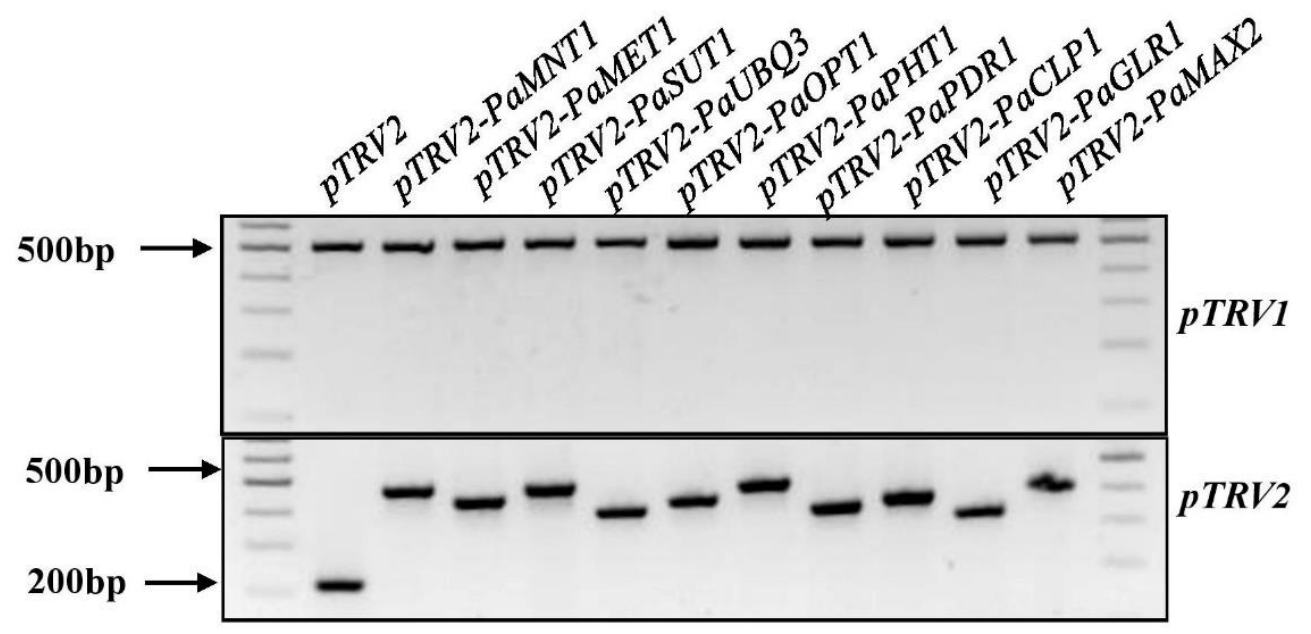

94

Fig. 1. (a) Schematic representation of pTRV2 vector design used for the silencing P.aegyptiaca gene(s).

(b) Expression of the $p T R V 1$ alone and $p T R V 2$ recombinant clones, in the VIGS-treated $N$. benthamiana plants. PCR analysis of genomic DNA isolated from the leaves of $N$. benthamiana, control plants infiltrated with the empty $p T R V 2$ vector, and from the different VIGS-treated plants.

After 30 days of agroinfiltration, the hosts plant N. benthamiana were assayed for resistance to P. aegyptiaca, which were pre-infected with the parasite seeds in the greenhouse. Total number of $P$. aegyptiaca tubercles and total weights of the tubercles more than $2 \mathrm{~mm}$ were determined on $p T R V 2: P a Y F G$ and $p T R V 2$ control plants. $p T R V 2: P a Y F G$ treated plants expressing the 
104 the total number of parasite tubercles grown on the host plant. However, the total weight of 105 tubercle, grown on host plant containing silencing vector against PaMNT1, PaMET1, PaSUT1, $106 P a U B Q 3, P a O P T 1$ and $P a C L P 1$ genes were found to be significantly decreased, as compared 107 to the control plants treated with only $p T R V 2$ vector (Fig. 2a and b).

a

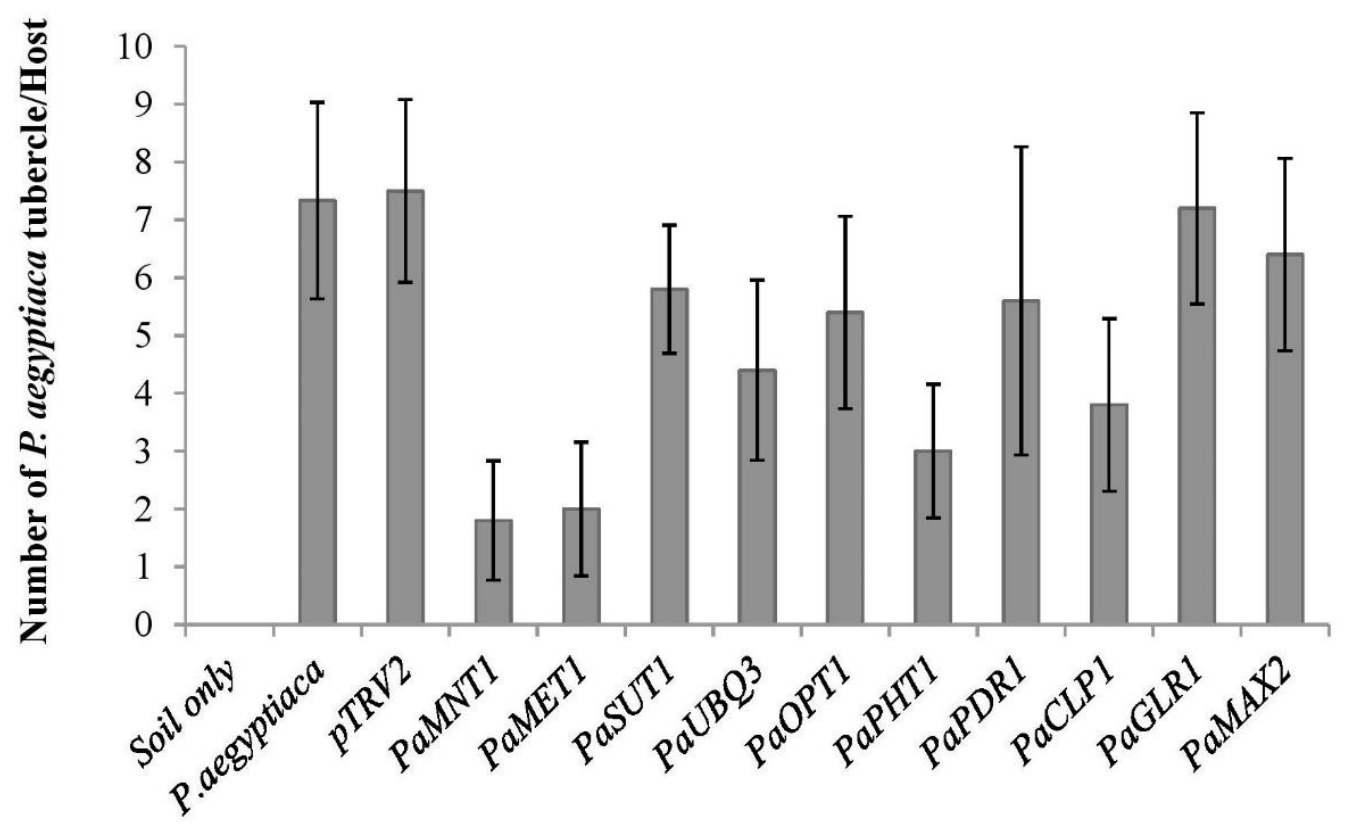

b

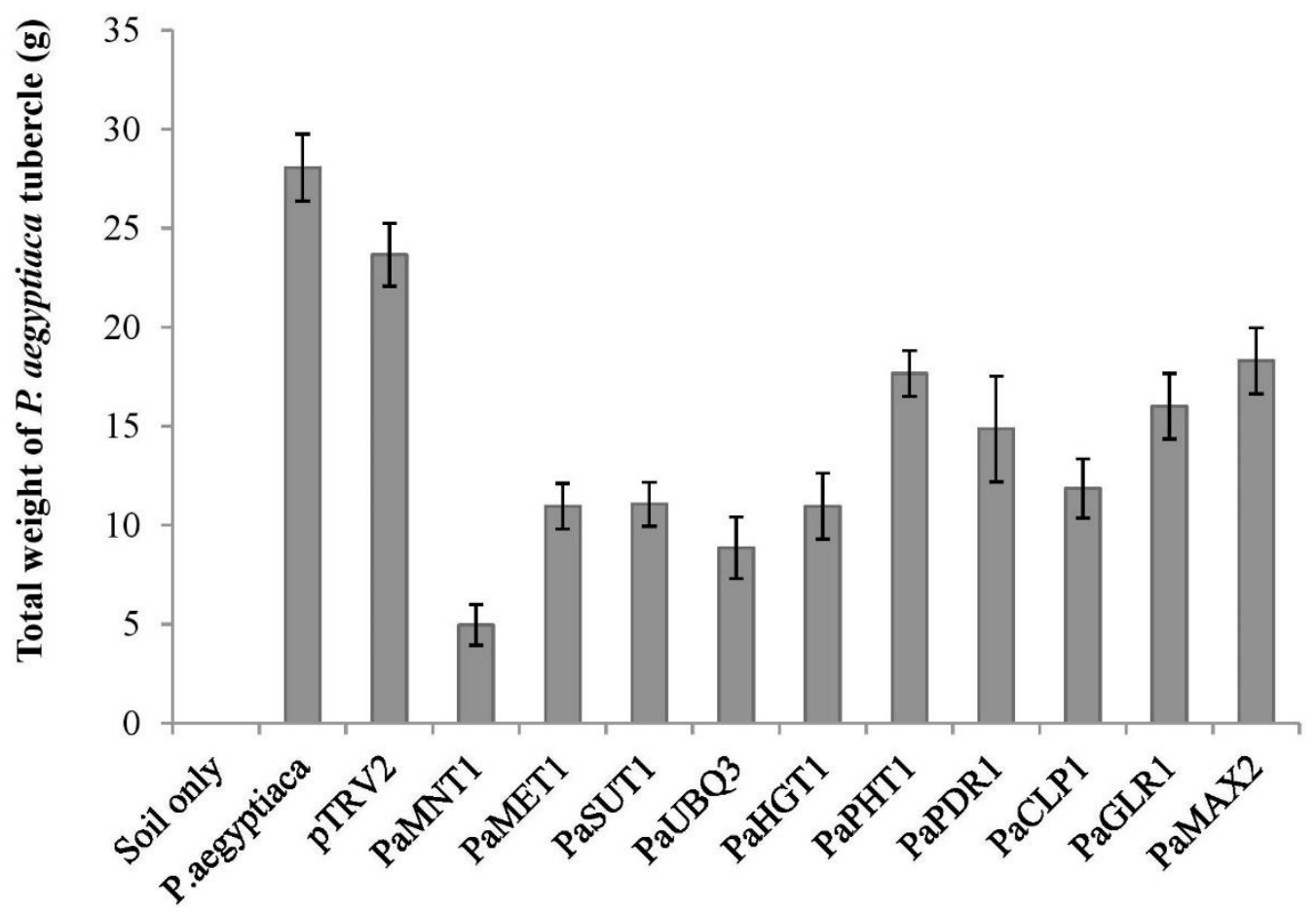


Fig. 2. Reduced growth of $P$. aegyptiaca tubercles grown on host root $N$. benthamiana. Total number of $P$. aegyptiaca tubercles attached to the host plants in the pot assay. Tubercles which were larger than

$1112 \mathrm{~mm}$ (diameter) counted and weighted. The number of total parasitic tubercles (a), total weight of 112 tubercles (b) was analysed. Bars represent average $\pm S E(n=5)$ value from three experiments with five 113 independent host plants.

114 Our results demonstrated that silencing of PaMNT1 consistently causes reduction in both, total number of tubercle and total weight of tubercle grown on the host root, so we analysed the transcript level of the targeted PaMNT1 grown on $N$. benthamiana host plants using

117 quantitative real time-PCR. The expression analysis data showed that the transcript levels of PaMNT1 were substantially reduced in the tubercles grown on host plants infected with recombinant $p T R V 2-P a M N T 1$ as compared to only the $p T R V 2$ vector. Moreover, viral coat protein expression was similar in both $p T R V 2$ empty vector and $p T R V 2-P a M N T 1$ treatments

(Fig. 3a and b). Results from our study strongly suggest that mannitol transport play an essential role in establishment of parasitic-host interaction and disruption of mannitol transporter could be used as a method to reduce the growth of parasitic weed P.aegyptiaca on host plants. 
a

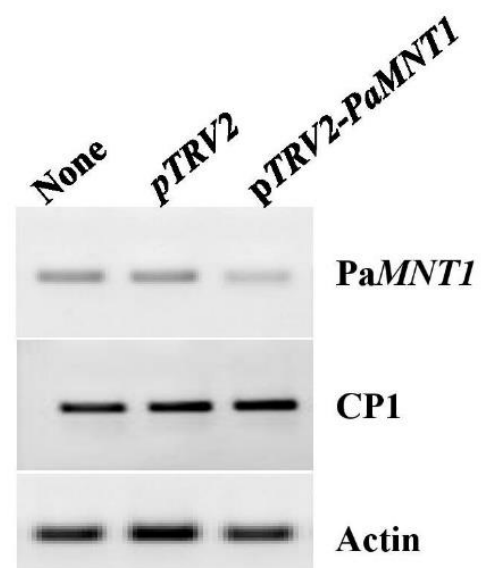

b

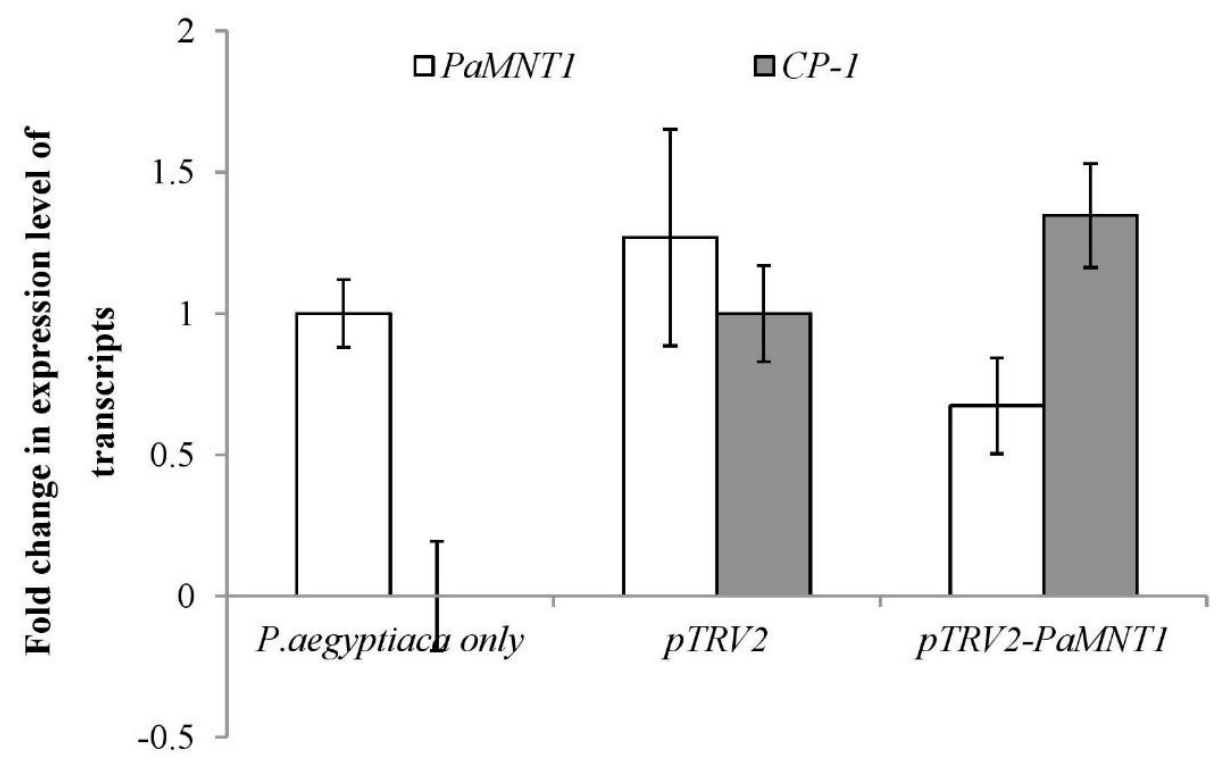

126 Fig. 3. Transcript level of PaMNT1, viral CP-1 in P. aegyptiaca tubercle. Expression of viral coat 127 transcript acts as control to measure the infection level $(\mathbf{a}, \mathbf{b})$. The actin gene served as internal control 128 for the expression. All analyses were performed using three biological replicates. Data presented as average $\pm \operatorname{SE}(n=3)$

\section{Discussion}

131 The complete removal of parasitic weeds from the agriculture field is the main problem in 132 growing agricultural crops which are susceptible to these types of parasitic weeds [14]. Root parasitic weeds, such as Phelipanche and Orobanche spp., are very challenging to control and 
several strategies adopted to limit these parasitic weeds have lower success rate [15]. Previous studies with $P$. aegyptiaca $C C D 7$ and $C C D 8$ genes suggested that silencing of these genes retarded the growth of parasite on the host [16]. Using a similar kind of approach, we reduced the transcript level of selected gene(s) involved in host-parasite interaction and demonstrated that the mannitol transporter gene could be important for survival of the plant parasite. Another study has reported that organisms under stress condition accumulate specific types of metabolites to a high extent such as organic sugars, polyols, amino acids, and other derivatives to regulate normal homeostasis of the cells by acting as osmotic stabilizers [17]. A similar observation has been widely accepted that mannitol protects cells against highly reactive oxygen species and also protects plants from salt and drought stress conditions[18, 19]. Our results are in consistent with the previous reports that Mannose 6-phosphate reductase (M6PR) enzyme which is involved in mannitol biosynthesis in plants having role in osmotic regulation of root specific parasites Orobanche minor during early phase of infection [20]. A recent study also reported that transient silencing of PaM6PR in $P$. aegyptiaca out of three genes (PaM6PR, $P a C W I$, and PaSUS1) involved in osmotic regulation process causes highest decrease in total amount of reducing sugars than the control, when grown on tomato host [21].

In this article, we have tested the role of several specific gene(s) of parasite $P$. aegyptiaca showing early upregulation after exposure to host root. From our results, we concluded that reduction in transcripts level of PaMNT1-mRNA in Phelipanche tubercles is directly correlated with reduction in mannitol transport from the host plant through the haustorium, which severely affects the development of Phelipanche on the host root and inhibiting or knocking mannitol biosynthesis or its transport could be used as a control strategy against these root specific parasitic weeds.

\section{Material and Methods:}


Putative P.aegyptiaca genes were selected for the VIGS. The complete sequences of P.ramosa

mannitol transporter, methionine synthase and sucrose transporter were selected from Uniprot and BLAST was performed against P.aegyptiaca using the PPGP website. For other candidate genes, Arabidopsis genome database (TAIR) was chosen as model and existence of homologous genes in P. aegyptiaca was confirmed using BLAST and reverse BLAST with the help of NCBI, sequence alignment was done using T-coffee and Clustal Omega software. The minimum E-value score with highly matched homologous sequences of respective candidate genes were selected.

\section{$p T R V 2$ vector construction and quantitative real time PCR}

The selected region for the gene of interest was amplified using the forward and reverse oligos flanking with restriction sites and cloned into a $p T R V 2$ vector using $B a m H I$ and $X b a \mathrm{I}$ restriction sites. The recombinant clones containing the insert genes were confirmed with diagnostics PCR and Sanger DNA sequencing analysis. Total RNA was extracted from 2 to $4 \mathrm{~mm}$ tubercles grown on silenced and unsilenced host plants using Spectrum-TM Plant Total RNA isolation

Kit (Sigma). Further the qRT-PCR analysis was performed in biological and experimental replicates using PCR Master Mix and calculation was done as described previously[22].

\section{Virus Induced Gene Silencing Assay}

Tobacco rattle virus mediated gene-silencing was performed as described previously [13] using the host $N$. benthamiana seedlings that had 6-8 leaves. In brief, the following plasmids: $p T R V 1$, pTRV2:PaYFG and empty pTRV2 were separately transformed into Agrobacterium tumefaciens strain EHA105 using electroporation and transformants were selected on rifampicin $(20 \mu \mathrm{g} / \mathrm{ml})$ and kanamycin $(50 \mu \mathrm{g} / \mathrm{ml})$ plate with $28^{\circ} \mathrm{C}$ incubation and positive transformants were confirmed by diagnostic PCR and DNA sequencing. To confirm the 
infection in host plants, genomic DNA is extracted from leaves and subjected to PCR using primer specific to $p T R V 1$ and $p T R V 2-P a Y F G$.

\section{Evaluation of Plant Resistance to the Parasite}

184 Host plants ( $N$. benthamiana) were used to study host-parasite interaction. Host plants were 185 germinated into 2-3 L pots filled with vermiculite culture soil and grown in a greenhouse with an average $16 \mathrm{~h}$ of daylight and a temperature of $24^{\circ} \mathrm{C}$. Grown seedlings of host plant transferred to 5-10L pots containing natural and vermiculite culture soil mixture were infested with $P$. aegyptiaca seeds (15 mg/kg soil). After 10 days agroinfiltration was performed on the lower side of host leaves. Host roots from VIGS and control treatments were collected 40-45 days after exposure to the $P$. aegyptiaca seeds and tubercles larger than $2 \mathrm{~mm}$ were collected for analysis.

Author Contributions: V.K.B designed the project. V.K.B. and J.A.N. carried out the experiments and collected data. V.K.B. drafted and finalized the manuscript. R.A. provided financial support for the study.

\section{Compliance with Ethical Standards:}

Disclosure of potential conflicts of interest: None

Informed consent: All the author has given consent to submit the manuscript. All authors declare no financial or non-financial conflict of interest.

\section{References:}

201 1. Mutuku, J.M., et al., Orobanchaceae parasite-host interactions. New Phytol, 2021. 230(1): p. 46-59. 
2. Westwood, J.H., et al., The evolution of parasitism in plants. Trends Plant Sci, 2010. 15(4): p. 227-35.

3. Wicke, S., et al., Mechanisms of functional and physical genome reduction in photosynthetic and nonphotosynthetic parasitic plants of the broomrape family. Plant Cell, 2013. 25(10): p. 3711-25.

4. Yoneyama, K., et al., Strigolactones: structures and biological activities. Pest Manag Sci, 2009. 65(5): p. 467-70.

5. Cardoso, C., C. Ruyter-Spira, and H.J. Bouwmeester, Strigolactones and root infestation by plant-parasitic Striga, Orobanche and Phelipanche spp. Plant Sci, 2011. 180(3): p. 414-20.

6. Kokla, A. and C.W. Melnyk, Developing a thief: Haustoria formation in parasitic plants. Dev Biol, 2018. 442(1): p. 53-59.

7. Yoshida, S., et al., The Haustorium, a Specialized Invasive Organ in Parasitic Plants. Annu Rev Plant Biol, 2016. 67: p. 643-67.

8. Smith, J.D., M.C. Mescher, and C.M. De Moraes, Implications of bioactive solute transfer from hosts to parasitic plants. Curr Opin Plant Biol, 2013. 16(4): p. 464-72.

9. Zwanenburg, B., A.S. Mwakaboko, and C. Kannan, Suicidal germination for parasitic weed control. Pest Manag Sci, 2016. 72(11): p. 2016-2025.

10. Yoder, J.I. and J.D. Scholes, Host plant resistance to parasitic weeds; recent progress and bottlenecks. Current Opinion in Plant Biology, 2010. 13(4): p. 478-484.

11. Aly, R., et al., Movement of protein and macromolecules between host plants and the parasitic weed Phelipanche aegyptiaca Pers. Plant Cell Rep, 2011. 30(12): p. 2233-41.

12. Clermont, K., et al., Comparative Metabolomics of Early Development of the Parasitic Plants Phelipanche aegyptiaca and Triphysaria versicolor. Metabolites, 2019. 9(6). 
13. Yang, Z., et al., Comparative transcriptome analyses reveal core parasitism genes and suggest gene duplication and repurposing as sources of structural novelty. Mol Biol Evol, 2015. 32(3): p. 767-90.

14. Bachan, S. and S.P. Dinesh-Kumar, Tobacco rattle virus (TRV)-based virus-induced gene silencing. Methods Mol Biol, 2012. 894: p. 83-92.

15. Fernandez-Aparicio, M., P. Delavault, and M.P. Timko, Management of Infection by Parasitic Weeds: A Review. Plants (Basel), 2020. 9(9).

16. Yoder, J.I. and J.D. Scholes, Host plant resistance to parasitic weeds; recent progress and bottlenecks. Curr Opin Plant Biol, 2010. 13(4): p. 478-84.

17. Dubey, N.K., et al., Enhanced Host-Parasite Resistance Based on Down-Regulation of Phelipanche aegyptiaca Target Genes Is Likely by Mobile Small RNA. Front Plant Sci, 2017. 8: p. 1574.

18. Aly, R., et al., Gene silencing of CCD7 and CCD8 in Phelipanche aegyptiaca by tobacco rattle virus system retarded the parasite development on the host. Plant Signal Behav, 2014. 9(8): p. e29376.

19. Bouwmeester, H.J., et al., Secondary metabolite signalling in host-parasitic plant interactions. Curr Opin Plant Biol, 2003. 6(4): p. 358-64.

20. Patel, T.K. and J.D. Williamson, Mannitol in Plants, Fungi, and Plant-Fungal Interactions. Trends Plant Sci, 2016. 21(6): p. 486-497.

21. Aly, R., et al., Gene silencing of mannose 6-phosphate reductase in the parasitic weed Orobanche aegyptiaca through the production of homologous dsRNA sequences in the host plant. Plant Biotechnol J, 2009. 7(6): p. 487-98.

22. Livak, K.J. and T.D. Schmittgen, Analysis of relative gene expression data using realtime quantitative PCR and the 2(-Delta Delta C(T)) Method. Methods, 2001. 25(4): p. 402-8. 